\title{
Inflammatory bowel disease in Bahrain: single-center experience
}

\section{Maheeba Abdulla' Mahmood Al Saeed' Rawdha Hameed Fardan' Hawra Faisal Alalwan' Zahra S Ali Almosawi' Amal Fuad Almahroos' Jehad Al Qamish²}

'Department of Internal Medicine, Salmaniya Medical Complex, Manama, Bahrain, ${ }^{2}$ Ibn Al Nafees Hospital, Manama, Bahrain
Correspondence: Maheeba Abdulla Department of Internal Medicine, Salmaniya Medical Complex, PO Box 12, Manama, Bahrain

Email amaheeba@hotmail.com
This article was published in the following Dove Press journal:

Clinical and Experimental Gastroenterology

14 July 2017

Number of times this article has been viewed

Purpose: The number of newly diagnosed inflammatory bowel disease (IBD) cases such as ulcerative colitis (UC), Crohn's disease (CD), and indeterminate colitis (IC) is rapidly increasing in Gulf countries and Saudi Arabia. The aim of this study was to investigate the incidence and prevalence of IBD in patients who have attended the Salmaniya Medical Complex, Bahrain, between the years 1984 and 2014.

Patients and methods: All patients who had attended the Salmaniya Medical Complex, Bahrain, and had been diagnosed with UC, CD, or IC, between the years 1984 and 2014, were included in the analysis. Data collected were: patient demographics, symptoms, clinical signs, complications, surgical interventions, extent of disease, endoscopic findings, histopathology, and lab measurements.

Results: A total of 187 cases were included; 61 had CD, 123 had UC, and a further 3 cases presented with IC. A clear increase in the incidence and prevalence of IBD can be seen in this cohort. The prevalence of IBD was calculated to be $26.25 / 10^{5}$ cases. The average number of IBD cases increased from 3 cases (average for the years 1984-2001) to 12 cases (average for the years 2002-2014). A number of factors correlate positively or negatively with CD and UC. In the current study, a link between gastrointestinal complications in CD cases and the use of steroids as a treatment was noted ( $p$-value -0.02 ). Age also had a significant influence on the need for surgery in CD cases ( $p$-value - 0.04 ), and a family history of UC was statistically linked to surgical intervention ( $p$-value -0.05 ).

Conclusions: IBD can no longer be considered a rare disease in Bahrain. The incidence of both UC and CD is steadily increasing. There is a need for increasing awareness of the Bahraini public to IBD in order for proper medical care to be given.

Keywords: inflammatory bowel diseases, Crohn's disease, ulcerative colitis, colon, gastrointestinal disorder, IBD, Bahrain

\section{Introduction}

Inflammatory bowel disease (IBD) is an umbrella term for a group of disorders in which the gastrointestinal (GI) tract becomes inflamed. Crohn's disease (CD) and ulcerative colitis (UC) represent the two main constituents of IBD and are triggered by a complex interaction of environmental exposure, genetic susceptibility, and dysregulated immune responses. ${ }^{1}$ Traditionally, IBD was believed to be a disorder of the Western world with the disease occurring most commonly in developed countries such as North America and Europe. ${ }^{2}$ However, in the past decade, there has been a rapid increase in the number of IBD cases reported in other regions such as Asia, Africa, and Latin America ${ }^{3}$. As such, the previously noted ethnic and racial differences appear to 
be narrowing. While the exact prevalence and incidence of IBD in these countries are still unknown, increased awareness and socioeconomic changes have allowed the etiology of IBD in these regions to be studied.

Both CD and UC are chronic, relapsing, inflammatory disorders mediated by the immune system. These diseases are more commonly diagnosed in adults; however, they can occur in children, and pediatric IBD cases now account for $7 \%-20 \%$ of all IBD cases. ${ }^{4} \mathrm{CD}$ can affect the whole GI tract, whereas UC affects the colonic mucosa. ${ }^{5,6}$ Certain environmental risk factors, such as smoking and appendectomy, have been shown to affect IBD occurrence. Interestingly, smoking has been reported to reduce the risk for UC while increasing the risk for $\mathrm{CD} .^{7-11}$ There also appears to be a slight gender difference as UC is known to occur more frequently in men (60\%), whereas CD is reported to occur up to $30 \%$ more frequently in women ${ }^{4}$.

The increased incidence and prevalence of IBD in the Middle East has been attributed to the westernization of lifestyles, including dietary and environmental changes. ${ }^{12-14}$ In Saudi Arabia and Kuwait, the annual rate of UC cases is reported to be in a steady-state, while the incidence of $\mathrm{CD}$ is rapidly increasing. ${ }^{12,15,16}$ In a retrospective study, the demographic and clinical data of 693 Saudi patients with IBD between the years 1993 and 2009 were described. The proportion of CD to UC patients in this study was seen to be similar to prior reports. ${ }^{17,18}$ The aim of this study is to report the occurrence of IBD in patients who presented to the Salmaniya Medical Complex, in Bahrain, over 3 decades (1984-2014), and to correlate the data with previously published findings.

\section{Ethical approval statement}

This study was reviewed and approved by the Salmaniya Medical Complex Institutional Review Board. Due to the patient data being anonymised and the retrospective nature of the study, written informed consent was waived by the Salmaniya Medical Complex Institutional Review Board.

\section{Patients and methods}

Male and female patients between 4 and 79 years, who had been diagnosed with UC, CD, or indeterminate colitis (IC) between the years 1984 and 2014, were included in the study. The patients presented to the Salmaniya Medical Complex, Bahrain, and all the data were extracted from the individual patient files. The diagnosis was established based on clinical history and examination, supported by lab investigation (inflammatory markers such as ESR and CRP, but without calprotectin or lactoferrin as both are not available in our hospital), endoscopy, and biopsy confirmation. A large body of data was collected for these patients including, but not limited to, 1) patients' demographics (age, gender, nationality, weight, height, body mass index [BMI], vaccination history, smoking and alcohol status, age at diagnosis, duration of disease, HIV status, and family history of IBD), 2) symptoms (abdominal pain, diarrhea, per rectal [PR] bleed, mucus, anorexia, weight loss, tenesmus, fever, perianal sacculitis, nausea/vomiting, mouth ulcer, joint pain, back pain, eye symptoms, and pruritis), 3) clinical presentation (aphthous ulcer, abdominal tenderness, abdominal mass, eye signs, skeletal, skin lesions, pallor, clubbing, fistula, perianal abscess, fissure, stenosis, and tags), 4) complications (anemia, intestinal obstruction, jaundice, sepsis, growth retardation, toxic megacolon, stricture, cancer, and operation), 5) surgical intervention (colectomy, stricturoplasty, fistulotomy, abscess drain, diversion, fistulectomy, stent placement, and small bowel obstruction), 6) extent of disease (Crohn's colitis, small bowel, ileocolonic, ileocecal, pancolitis, proctosigmoiditis left, and proctitis), 7) lab measurements, 8) endoscopic findings (colon and upper GI tract), 9) histopathology (granulation, microscopic, dysplasia, collagenous colitis, and carcinoma), 10) medications (salazopyrine, 5-aminosalicylates [5-ASA], corticosteroids, azathioprine, 6-mercaptopurine, cyclosporine, infliximab, and adalimumab [Humira]), and 11) further complications.

Data are presented as mean \pm standard deviation for continuous variables and percentages for categorical variables. The difference between groups was analyzed by Student's $t$-test for continuous variables and by chi-square test for categorical variables.

\section{Results}

\section{Patient demographics}

A total of 187 male and female cases were included in the study; of these, 61 had CD, 123 had UC, and a further 3 cases presented with IC. Of the $61 \mathrm{CD}$ cases, 36 were males $(59 \%)$ and 25 were females $(41 \%)$. Of the total 123 cases of UC, 65 were males $(52.85 \%)$ and 58 were females $(47.15 \%)$, while 1 male (33\%) and 2 females (66\%) had a diagnosis of IC (Table 1). The average height, weight, and BMI were calculated for a portion of the cohort. Overall, the mean height of IBD cases was $133.2 \mathrm{~cm}(\mathrm{n}=15)$, the mean weight 
Table I Patient demographics

\begin{tabular}{|c|c|c|c|c|}
\hline & $\begin{array}{l}\text { Crohn's disease } \\
(\mathrm{N}=6 \mathrm{I})\end{array}$ & $\begin{array}{l}\text { Ulcerative } \\
\text { colitis }(N=123)\end{array}$ & $\begin{array}{l}\text { Indeterminant } \\
\text { colitis }(\mathbf{N}=3)\end{array}$ & $\begin{array}{l}\text { IBD total } \\
(n=187)\end{array}$ \\
\hline \multicolumn{5}{|l|}{ Nationality } \\
\hline Bahraini & $\mathrm{N}=59$ & $\mathrm{~N}=102$ & $N=I$ & $N=162$ \\
\hline NonBahraini & $\mathrm{N}=2$ & $\mathrm{~N}=2 \mathrm{I}$ & $\mathrm{N}=2$ & $\mathrm{~N}=25$ \\
\hline \multicolumn{5}{|c|}{ Age at diagnosis (years) } \\
\hline Mean (sample size) & $24.13(n=60)$ & $28.35(n=|2|)$ & $32.33(n=3)$ & $27.03(n=184)$ \\
\hline \multicolumn{5}{|l|}{ Gender, n (\%) } \\
\hline Male & $36(59)$ & $65(52.85)$ & I (33) & $102(54.55)$ \\
\hline Female & $25(4 I)$ & $58(47.15)$ & $2(66)$ & $85(45.45)$ \\
\hline \multicolumn{5}{|l|}{ Height (cm) } \\
\hline Mean & $|38.7|(n=7)$ & $128.38(n=8)$ & No data & $133.2(n=\mid 5)$ \\
\hline \multicolumn{5}{|l|}{ Weight (kg) } \\
\hline Mean & $38.14(n=20)$ & $51.94(n=40)$ & $57(n=I)$ & $47.50(n=61)$ \\
\hline \multicolumn{5}{|l|}{ BMI $\left(\mathrm{kg} / \mathrm{m}^{2}\right)$} \\
\hline Mean & $16.53(n=7)$ & $18.22(n=6)$ & No data & $|7.3|(n=13)$ \\
\hline \multicolumn{5}{|l|}{ Smoker } \\
\hline Yes & $14.8 \%$ & $7.3 \%$ & 0 & $9.6 \%$ \\
\hline \multicolumn{5}{|l|}{ Alcohol } \\
\hline Yes & $1.6 \%$ & $4.1 \%$ & 0 & $3.7 \%$ \\
\hline
\end{tabular}

Abbreviations: BMI, body mass index; IBD, inflammatory bowel disease.

was $47.50 \mathrm{~kg}(\mathrm{n}=61)$, and the mean BMI was calculated at $17.31 \mathrm{~kg} / \mathrm{m}^{2}(\mathrm{n}=13)$. Overall, $9.6 \%$ of IBD patients in the Bahrain cohort were smokers, while $3.7 \%$ consumed alcohol (Table 1).

\section{Incidence of IBD is increasing in the Bahrain region}

In this study, the total number of UC, CD, or IC cases, reported between the years 1984 and 2014, was recorded. Considering the average population of Bahrain at this time (712,381), the total prevalence of IBD reported to the Salmaniya Medical Complex was calculated to be $\sim 26.25 / 10^{5}$ cases. The prevalence came to $8.56 / 10^{5}$ cases for $\mathrm{CD}, 17.27 / 10^{5}$ cases for UC, and $0.42 / 10^{5}$ cases for IC (Figure 1). The average number of IBD cases reported between the years 1984 and 2001 was 3 . This increased to an average of 12 cases per year between the years 2002 and 2014. In the case of CD, the average number of annual cases was 0.4 between the years 1984 and 2001 , and this number increased to an average of 4 cases per year between 2002 and 2014. For UC, the average number of cases between 1984 and 2001 was 2, while this increased to 8 for the years 2002-2014. Therefore, there was an increase of at least 4 times as many cases reported in the later years in comparison with the earlier years.

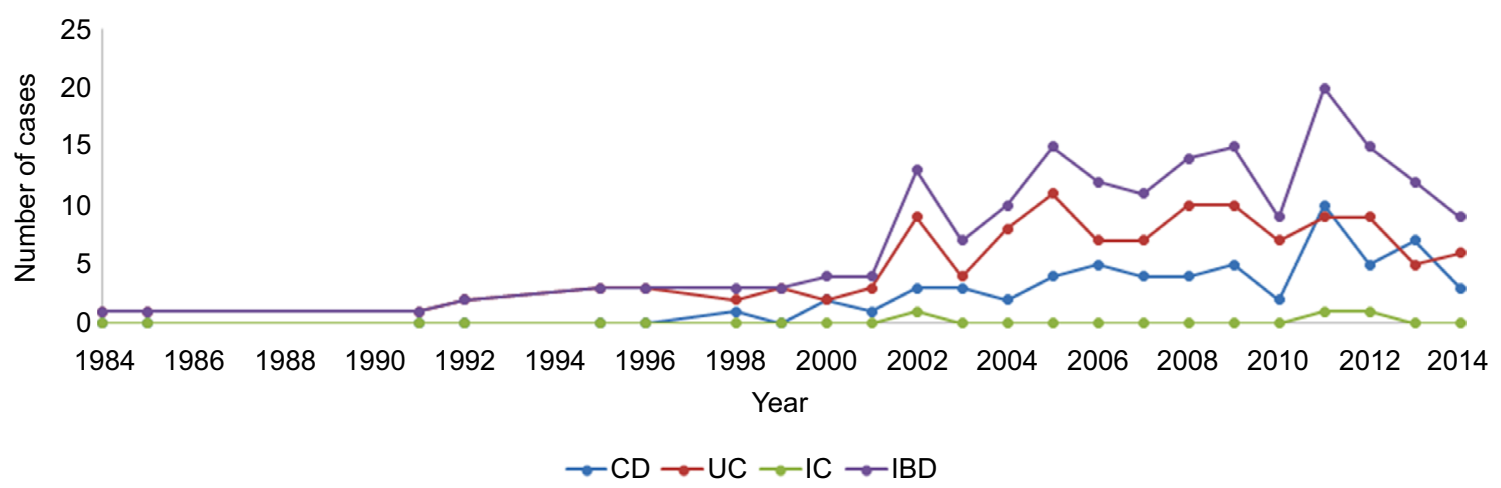

Figure I Incidence of IBD is increasing with time in the Bahrain region.

Notes: The number of cases of CD and UC of IBD cases, reported between 1984 and 2014, are shown above. There is a clear overall increase in the number of reported cases of $C D$ and $U C$.

Abbreviations: CD, Crohn's disease; UC, ulcerative colitis; IC, indeterminate colitis; IBD, inflammatory bowel disease. 


\section{Risk factors for serious outcomes for CD and UC in Bahrain}

A number of factors correlate positively or negatively with CD and UC. In this study, a number of these factors were analyzed against 3 variables: anemia, GI complications, and surgical intervention. The factors that correlate positively with serious outcomes in CD patients that were identified include: 1) younger age, 2) perianal symptoms, 3) steroid use, 4) current smoking, and 5) extensive disease. The factor identified that negatively correlates with serious outcomes in $\mathrm{CD}$ is a family history of CD. For UC, younger age (correlates positively with serious outcomes) was identified, and extra-intestinal manifestations, as well as a family history of UC, which correlates negatively with serious outcomes in UC, were identified (Table 2). Although not quite reaching a significance of $p<0.05, \mathrm{CD}$ patients with anemia appeared more likely to have perianal symptoms than patients who did not have anemia ( $p$-value -0.06 ). In addition, if a patient with CD had GI complications, they were statistically more likely to need steroids as a treatment ( $p$-value -0.02$)$. Age also had a significant influence on the need for surgery in CD patients ( $p$-value -0.04 ). Furthermore, a family history of UC was statistically linked to surgical intervention ( $p$-value -0.05 ) and trended, but did not reach significance, for a link between anemia and family history in UC patients ( $p$-values -0.06$)$.

\section{Percentage of men affected by IBD is increasing over time}

The annual trends in gender distribution were also investigated. Analysis of the percentage of male cases of $\mathrm{CD}$, $\mathrm{UC}$, and IBD, as compared with the total number of cases, suggests that the percentage of these disorders in men is increasing over time (Figure S1). In particular, CD appears to be becoming more common in men (Figure S1A), whereas $\mathrm{UC}$ is following the same trend, but the effect is not as prominent (Figure S1B).

\section{The average numbers of hospital admissions are decreasing with time}

The annual trends in the number of hospital admissions for CD, UC, and IBD during 1984-2014 appear to follow a slightly decreasing trend over time. The average number of hospital admissions for $\mathrm{CD}$ was $3.3 \pm 2.4$, and for $\mathrm{UC}$ was $2.9 \pm 2.3$ (Figure S2).

\section{The percentage occurrence of covariates with UC, CD, and IBD}

Covariates were also investigated. These included family history, HIV, hypertension, diabetes mellitus, alcohol, smoking status, and vaccination history. Approximately, $10 \%$ of IBD patients had a family history of the disorder (UC; 9\%, CD; $13 \%$ ), and $10 \%$ were smokers (UC; 7\%, CD 15\%; Figure S3). Overall, $28 \%$ of IBD patients were also vaccinated; $39 \%$ of $\mathrm{CD}$, and $22 \%$ of UC patients.

Abdominal pain was the main symptom in $77 \%$ of UC patients and $95 \%$ of CD patients. Diarrhea also occurred in a high percentage of cases, $81 \%$ of UC and $52 \%$ of CD cases (Figure S4A). PR bleeds occurred mainly in UC patients with an occurrence of $69 \%$ in comparison with $33 \%$ of $C D$ patients (Figure S4A). Weight loss and nausea/vomiting also occurred at a high frequency in IBD patients, affecting nearly $50 \%$. From a clinical perspective, abdominal tenderness is reported to be the clinical sign with the highest percentage

Table 2 Risk factors for serious outcomes for Crohn's disease and ulcerative colitis in Bahrain

\begin{tabular}{|c|c|c|c|c|c|c|c|c|c|}
\hline \multirow[t]{3}{*}{ Factor (yes/no) } & \multicolumn{9}{|c|}{ Outcomes (level of significance; yes vs. no) } \\
\hline & \multicolumn{3}{|l|}{ Anemia } & \multicolumn{3}{|c|}{ Gastrointestinal complications } & \multicolumn{3}{|c|}{ Surgical intervention } \\
\hline & Yes & No & $p$-value & Yes & No & $P$-value & Yes & No & $p$-value \\
\hline \multicolumn{10}{|l|}{ Crohn's disease } \\
\hline Age (years) & $22.73 \pm 11.27$ & $26.81 \pm 11.15$ & 0.18 & $24.06 \pm 11.22$ & $24.22 \pm 11.63$ & 0.96 & $28.09 \pm 12.25$ & $21.90 \pm 10.23$ & 0.04 \\
\hline Perianal symptoms & $6 / 34$ & $0 / 21$ & 0.06 & $5 / 29$ & $1 / 26$ & 0.15 & $3 / 19$ & $3 / 36$ & 0.45 \\
\hline Need for steroids & $29 / 11$ & $12 / 9$ & 0.23 & $27 / 7$ & $14 / 13$ & 0.02 & $15 / 7$ & $26 / 13$ & 0.90 \\
\hline Current smoking & $8 / 32$ & $\mathrm{I} / 20$ & 0.11 & $6 / 28$ & $3 / 24$ & 0.47 & $3 / 19$ & $6 / 33$ & 0.85 \\
\hline Extensive disease & $1 / 39$ & $\mathrm{I} / 20$ & 0.64 & $1 / 33$ & $1 / 26$ & 0.95 & $|/ 2|$ & $1 / 38$ & 0.68 \\
\hline Family history & $6 / 34$ & $2 / 19$ & 0.55 & $7 / 27$ & $1 / 26$ & 0.05 & $3 / 19$ & $5 / 34$ & 0.93 \\
\hline \multicolumn{10}{|l|}{ Ulcerative colitis } \\
\hline Age (years) & $24.78 \pm 13.33$ & $32.71 \pm 16.77$ & 0.00 & $25.63 \pm|7.4|$ & $30.18 \pm 13.78$ & 0.11 & $24.50 \pm 11.94$ & $29.11 \pm 15.97$ & 0.22 \\
\hline $\begin{array}{l}\text { Extraintestinal } \\
\text { manifestation }\end{array}$ & $55 / 12$ & $50 / 6$ & 0.26 & $45 / 4$ & $60 / 14$ & 0.10 & $16 / 4$ & $89 / 14$ & 0.56 \\
\hline Family history & $9 / 58$ & $2 / 54$ & 0.06 & $5 / 44$ & $6 / 68$ & 0.69 & $1 / 19$ & $10 / 93$ & 0.50 \\
\hline
\end{tabular}

Note: Data presented as mean \pm standard deviation. 
occurrence in $\mathrm{CD}, \mathrm{UC}$, and IBD (43\% of CD and 59\% of CD cases) (Figure $\mathrm{S} 4 \mathrm{~B}$ ). All other clinical signs such as tags, stenosis, fissure, fistula, and so on, occurred at a relatively low frequency.

\section{Analysis of complications and surgical interventions}

The intestinal complications (vs. extraintestinal) and surgical interventions are detailed in Figure S5A. Complications such as cancer, stricture, toxic megacolon, growth retardation, operation, sepsis, jaundice, intestinal obstruction, and anemia were also analyzed (Figure S5B). Anemia was found to occur with the highest frequency in UC (54\%) and CD (66\%). Surgical interventions were also recorded. Colectomy was the most common surgical intervention, occurring in $13 \%$ of UC and CD cases. Abscess draining procedures were also required for $13 \%$ of $\mathrm{CD}$ cases (Figure $\mathrm{S} 5 \mathrm{C}$ ).

\section{Extent of disease and medications taken}

The extent of disease, as well as the most common drug treatments taken by IBD patients in Bahrain, was also recorded. Different parts of small bowel involvement, particularly the terminal, were seen in $48 \%$ of CD patients. Crohn's colitis, though less common, was diagnosed in $34 \%$ of CD patients. Pancolitis and proctitis were much rarer in CD patients ( $10 \%$ and $5 \%$, respectively). Pancolitis and proctitis were far more common in UC patients ( $47 \%$ and $30 \%$, respectively; Figure S6A).

Of the drug treatments taken, azathioprine (75\%), steroids $(67 \%)$, and 5-ASAs (90\%) were the most commonly used drug treatments for IBD at the Salmaniya Medical Complex in Bahrain (Figure S6B).

Biopsies were taken from all the parts of the colon including: terminal ileum, cecum, transverse colon, transverse colon, descending colon, sigmoid, and rectum. The histological evaluation of these samples revealed microscopic inflammation in $92 \%$ of UC and $82 \%$ of CD patients, representing the most common histological finding in IBD (Figure S7). Furthermore, granulation was seen to occur at a much higher frequency in $\mathrm{CD}$ patients $(51 \%)$ in comparison with UC patients (1\%), while dysplasia, collagenous colitis, and carcinoma were rare, or not present in these IBD samples.

\section{Occurrence of extraintestinal manifestations}

Extraintestinal manifestations of IBD are prevalent in both CD and UC patients and can involve almost any system, including the hepato-pancreatobiliary, renal, musculoskeletal, ocular, dermatologic, and pulmonary systems. The extraintestinal manifestations investigated in the current cohort included gallstones, primary sclerosing cholangitis, uveitis, episclerotitis, arthritis, ankylosing spondylitis, vasculitis, pyoderma gangrenosum, and erythema nodosum. Arthritis occurred in 5\% of IBD patients, and primary sclerosing cholangitis occurred in 5\% of UC patients. All other extraintestinal manifestations occurred at a frequency lower than 5\% (Figure S8).

\section{Discussion}

IBD was once described as a rare or nonexistent disease in Saudi Arabia and neighboring countries, but this is no longer the case. ${ }^{17,18}$ A recent study by Al-Mofarreh and AlMofleh, in which 693 patients were reviewed over 17 years, highlighted that UC and CD can no longer be considered as "rare". In their study, they revealed a steady-state incidence of UC patients, while there was a marked increase in the number of CD cases. ${ }^{12}$ In this study, the number of UC cases outweighs the number of CD cases (123 UC and $63 \mathrm{CD}$ ). This is a common trend around the world as the incidence of UC is normally reported to be higher than CD, except for in Canada, Australia, and some parts of the United States. ${ }^{4,12}$ In a review summarizing the global epidemiology of IBD, the incidence of UC was reported to be $0.6-24.3 / 100,000$ in Europe, 0.1-6.3/100,000 in Asia and the Middle East, and 0-19.2/100,000 in North America for the years 1930-2008. In the same study, the incidence of $\mathrm{CD}$ was reported as 0.3-12.7/100,000 in Europe, 0.04-5.0/100,000 in Asia and the Middle East, and 0-20.2/100,000 in North America. ${ }^{19}$ The evolution of IBD in countries undergoing westernization has been described as follows: an initial low IBD incidence is followed by an increase in UC while CD remains low, this is then followed by an increase in $\mathrm{CD}$ cases which slowly approach the levels of $\mathrm{UC}$ incidences. ${ }^{4}$ The current Bahrain cohort of IBD patients appears to be following this general pattern of disease development and displays similar trends to Japan, South Korea, Singapore, and Saudi Arabia. 5,20

Investigation of the annual trends of incidence during the course of this study (1984-2014) revealed that both UC and $\mathrm{CD}$ appear to be increasing at a similar rate. Interestingly, $\mathrm{CD}$ appears to be becoming more common in men in Bahrain than in women. There was also a similar trend for UC; however, this was not as prominent. There have been some previous reports that there is a slight predominance of $\mathrm{CD}$ in women ${ }^{4,21,22}$; however, this is not always the case. ${ }^{23}$ Social and cultural beliefs in this part of the world may explain the apparent increasing incidence in males, as females may be 
less likely to attend a hospital for certain IBD symptoms. This may not be the case in Bahrain, as males and females equally seek medical advice. In addition, medical services are available across the country and are accessible to everybody.

As expected, small bowel involvement and Crohn's colitis were seen to occur at a high frequency in CD cases in the current Bahrain cohort. Similarly, UC patients were found to have a high percentage occurrence of pancolitis and proctitis. Endoscopic data also revealed colon inflammation in over $87 \%$ of cases.

Of the drug treatments taken, 5-ASA was revealed to be the most commonly used treatment for IBD in Bahrain, followed by azathioprine and steroids. During the time frame of this study (1984-2001), adalimumab use was restricted to younger patients with moderate nonfistulizing disease. As such, only $16 \%$ of IBD patients were on adalimumab and $20 \%$ on infliximab. The advent of biologics has been described as a new era for IBD management. ${ }^{24}$ Currently, anti-TNF therapy such as adalimumab and infliximab is primarily suitable for the treatment of moderate-to-severe UC and CD. Vedolizumab, recently approved for use in UC patients, may also be beneficial for $\mathrm{CD}$ patients who have not responded to other anti-TNF agents in the past. ${ }^{25}$ In this study, more than $50 \%$ of patients were anemic. GI complications also occurred at a very high frequency as more than $40 \%$ patients were affected. In addition, surgical intervention was necessary in $16 \%$ of UC patients and $36 \%$ of CD patients. The presence of 1 or more of these 3 variables was also found to have an impact on the disease course. For example, an anemic CD patient appeared to be more likely to have perianal symptoms than a CD patient without anemia. Although this link did not quite reach significance, it likely would have if a larger sample size were used. It was also discovered that $\mathrm{CD}$ patients presenting with GI complications were statistically more likely to need steroids as a treatment. Furthermore, age also had a significant influence on the need for surgery in CD patients, while a family history of UC increased the likelihood that surgical intervention would be required.

\section{Conclusion}

IBD can no longer be considered a rare disease in Bahrain. The incidence of both UC and CD is steadily increasing over time, and the latter appears to be more common in men than women in Bahrain. The dramatic increase in the prevalence and incidence of IBD in Bahrain and neighboring countries highlights these disorders as one of the major emerging diseases in newly westernized countries. Efforts will need to be made to inform and educate the public on signs and symptoms of IBD. In addition, further research by clinicians in the region is needed to thoroughly investigate the epidemiology of these disorders.

\section{Acknowledgments}

We would like to acknowledge the medical writing, language editing, proofreading, and editorial assistance of ResearchMedics in the preparation of this manuscript.

\section{Author contributions}

MA, MAS, RHF, HFA, ZSAA, AFA, and JAQ were involved in writing the proposal, conception, and design of the study, analysis and interpretation of data, critical revision of the manuscript, and approval of the final draft.

\section{Disclosure}

The authors report no conflicts of interest in this work.

\section{References}

1. Hanauer SB. Inflammatory bowel disease: epidemiology, pathogenesis, and therapeutic opportunities. Inflamm Bowel Dis. 2006;12(Suppl 1): S3-S9.

2. Ng WK, Wong SH, Ng SC. Changing epidemiological trends of inflammatory bowel disease in Asia. Intest Res. 2016;14(2):111-119.

3. Loftus EV Jr. Clinical epidemiology of inflammatory bowel disease: incidence, prevalence, and environmental influences. Gastroenterology. 2004;126(6):1504-1517.

4. Cosnes J, Gower-Rousseau C, Seksik P, Cortot A. Epidemiology and natural history of inflammatory bowel diseases. Gastroenterology. 2011;140(6):1785-1794.

5. Khawaja AQ, Sawan AS. Inflammatory bowel disease in the Western Saudi Arabia. Saudi Med J. 2009;30(4):537-540.

6. Sartir RB. Mechanisms of disease: pathogenesis of Crohn's disease and ulcerative colitis. Nat Clin Pract Gastroenterol Hepatol. 2006;3(7):390-407.

7. Legaki E, Gazouli M. Influence of environmental factors in the development of inflammatory bowel diseases. World J Gastrointest Pharmacol Ther. 2016;7(1):112-125.

8. Tuvlin JA, Raza SS, Bracamonte S, et al. Smoking and inflammatory bowel disease: trends in familial and sporadic cohorts. Inflamm Bowel Dis. 2007;13(5):573-579.

9. Reese GE, Nanidis T, Borysiewicz C, Yamamoto T, Orchard T, Tekkis PP. The effect of smoking after surgery for Crohn's disease: a metaanalysis of observational studies. Int J Colorectal Dis. 2008;23(12): 1213-1221.

10. Cosnes J. Tobacco and IBD: relevance in the understanding of disease mechanisms and clinical practice. Best Pract Res Clin Gastroenterol. 2004;18(3):481-496.

11. Koutroubakis IE, Vlachonikolis IG, Kouroumalis EA. Role of appendicitis and appendectomy in the pathogenesis of ulcerative colitis: a critical review. Inflamm Bowel Dis. 2002;8(4):277-286.

12. Al-Mofarreh MA, Al-Mofleh IA. Emerging inflammatory bowel disease in saudi outpatients: a report of 693 cases. Saudi J Gastroenterol. 2013;19(1):16-22.

13. Prideaux L, Kamm MA, De Cruz PP, Chan FK, Ng SC. Inflammatory bowel disease in Asia: a systematic review. $J$ Gastroenterol Hepatol. 2012;27(8):1266-1280.

14. Molodecky NA, Soon IS, Rabi DM, et al. Increasing incidence and prevalence of the inflammatory bowel diseases with time, based on systematic review. Gastroenterology. 2012;142(1):46-54.e42. 
15. Al-Ghamdi AS, Al-Mofleh IA, Al-Rashed RS, et al. Epidemiology and outcome of Crohn's disease in a teaching hospital in Riyadh. World $J$ Gastroenterol. 2004;10(9):1341-1344.

16. Al-Qabandi WA, Buhamrah EK, Hamadi KA, Al-Osaimi SA, AlRuwayeh AA, Madda J. Inflammatory bowel disease in children, an evolving problem in Kuwait. Saudi J Gastroenterol. 2011;17(5): 323-327.

17. Fadda MA, Peedikayil MC, Kagevi I, et al. Inflammatory bowel disease in Saudi Arabia: a hospital-based clinical study of 312 patients. Ann Saudi Med. 2012;32(3):276-282.

18. Siddique I, Alazmi W, Al-Ali J, et al. Clinical epidemiology of Crohn's disease in Arabs based on the Montreal Classification. Inflamm Bowel Dis. 2012;18(9):1689-1697.

19. M'Koma AE. Inflammatory bowel disease: an expanding global health problem. Clin Med Insights Gastroenterol. 2013;6:33-47.

20. Ahuja V, Tandon RK. Inflammatory bowel disease in the Asia-Pacific area: a comparison with developed countries and regional differences. J Dig Dis. 2010;11(3):134-147.
21. Bernstein CN, Blanchard JF, Rawsthorne P, Wajda A. Epidemiology of Crohn's disease and ulcerative colitis in a central Canadian province: a population-based study. Am J Epidemiol. 1999;149(10):916-924.

22. Moum B, Vatn MH, Ekbom A, Aadland E, Fausa O, Lygren I, Stray N, Sauar J, Schulz T. Incidence of Crohn's disease in four counties in southeastern Norway, 1990-93. A prospective population-based study. The Inflammatory Bowel South-Eastern Norway (IBSEN) Study Group of Gastroenterologists. Scand J Gastroenterol. 1996;31(4): 355-361.

23. Jussila A, Virta LJ, Kautiainen H, Rekiaro M, Nieminen U, Färkkilä MA. Increasing incidence of inflammatory bowel diseases between 2000 and 2007: a nationwide register study in Finland. Inflamm Bowel Dis. 2012;18(3):555-561.

24. Mao R, Hu PJ. The future of IBD therapy: where are we and where should we go next? Dig Dis. 2016;34(1-2):175-179.

25. Sands BE, Feagan BG, Rutgeerts $P$, et al. Effects of vedolizumab induction therapy for patients with Crohn's disease in whom tumor necrosis factor antagonist treatment failed. Gastroenterology. 2014;147(3):618-627. 


\section{Supplementary materials}

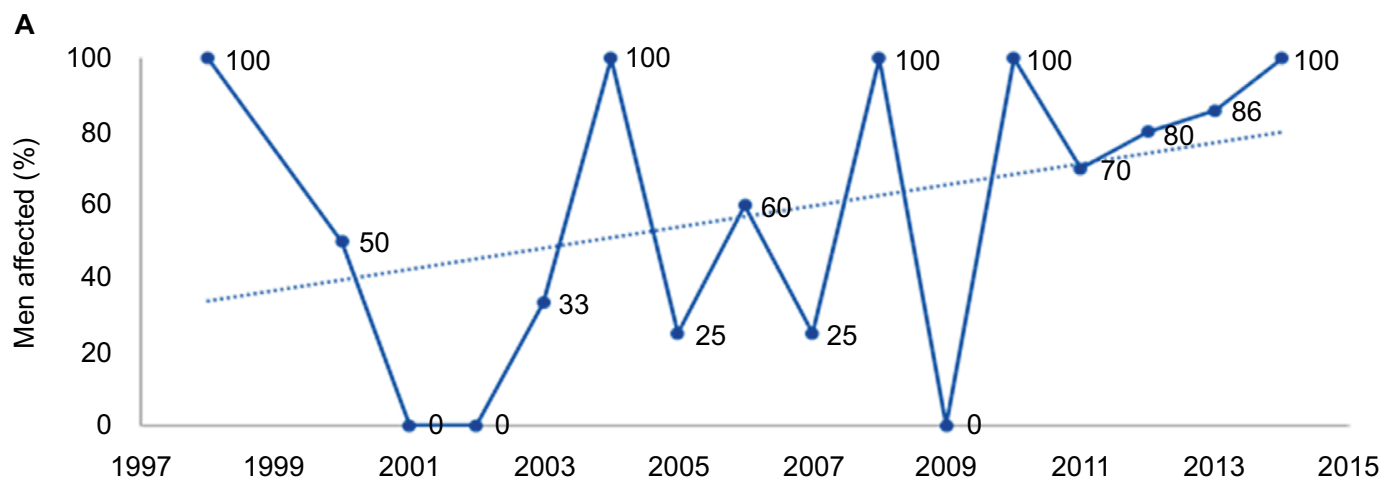

B

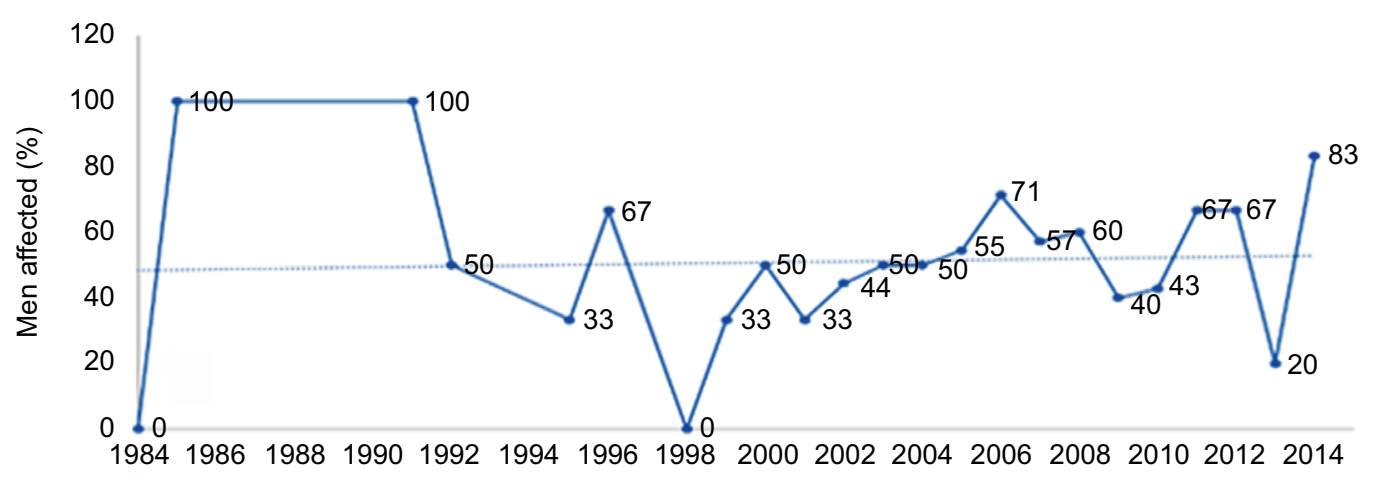

C

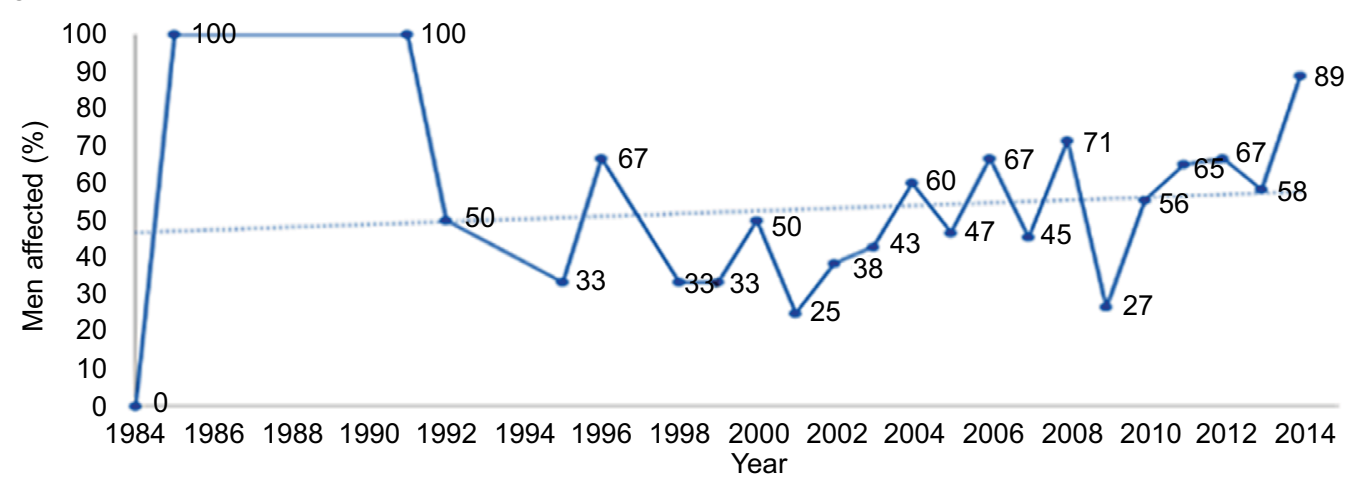

Figure SI The percentage of men affected with CD, UC, and IBD, as compared to the total number of cases between 1984-20I4, are depicted in A, B, and C, respectively. Notes: (A) The percentage of men affected with $C D$ appears to be increasing over time. (B) The percentage of $U C$ cases in men is also trending toward an increase although to a lesser extent than with CD. (C) The combined total number of IBD cases, therefore, also indicates an increase in the percentage of men reporting IBD.

Abbreviations: CD, Crohn's disease; UC, ulcerative colitis; IBD, inflammatory bowel disease. 
A

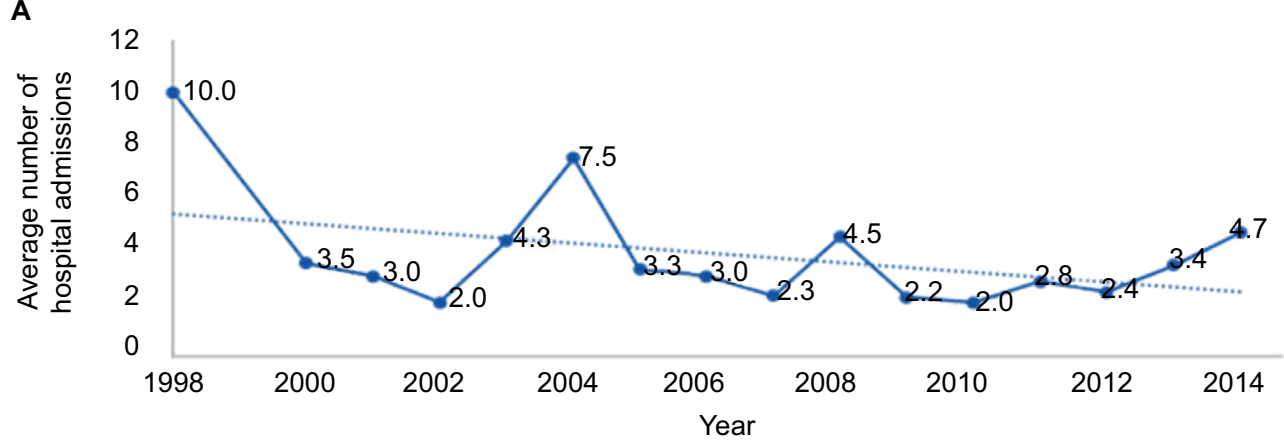

B

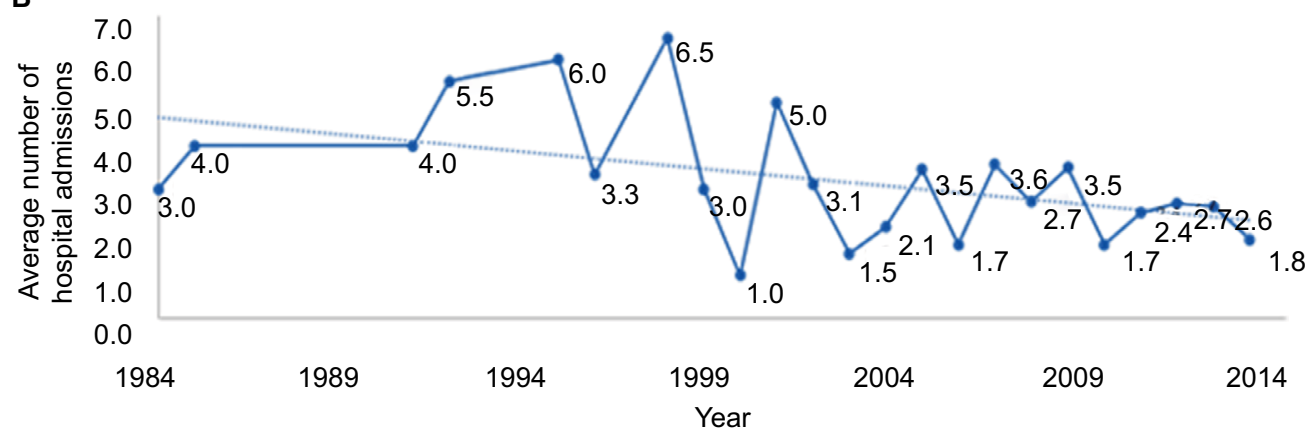

C

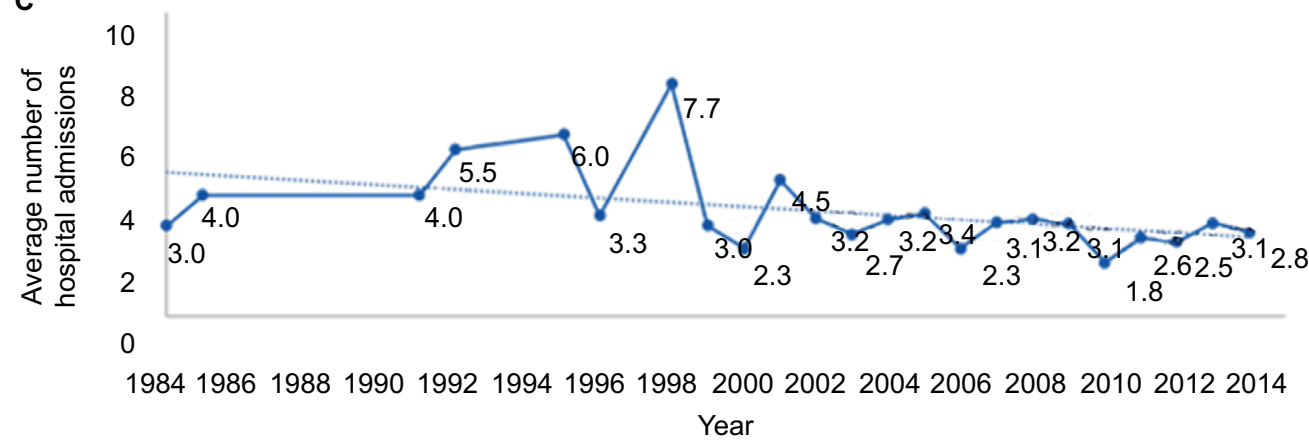

Figure S2 The annual trends in the number of hospital admissions for CD, UC, and IBD between 1984 and 2014 are depicted above in A, B, and C, respectively. Notes: The average number of hospital admissions for CD (A), UC (B), and IBD (C) appear to follow a slightly decreasing trend over time.

Abbreviations: CD, Crohn's disease; UC, ulcerative colitis; IBD, inflammatory bowel disease. 


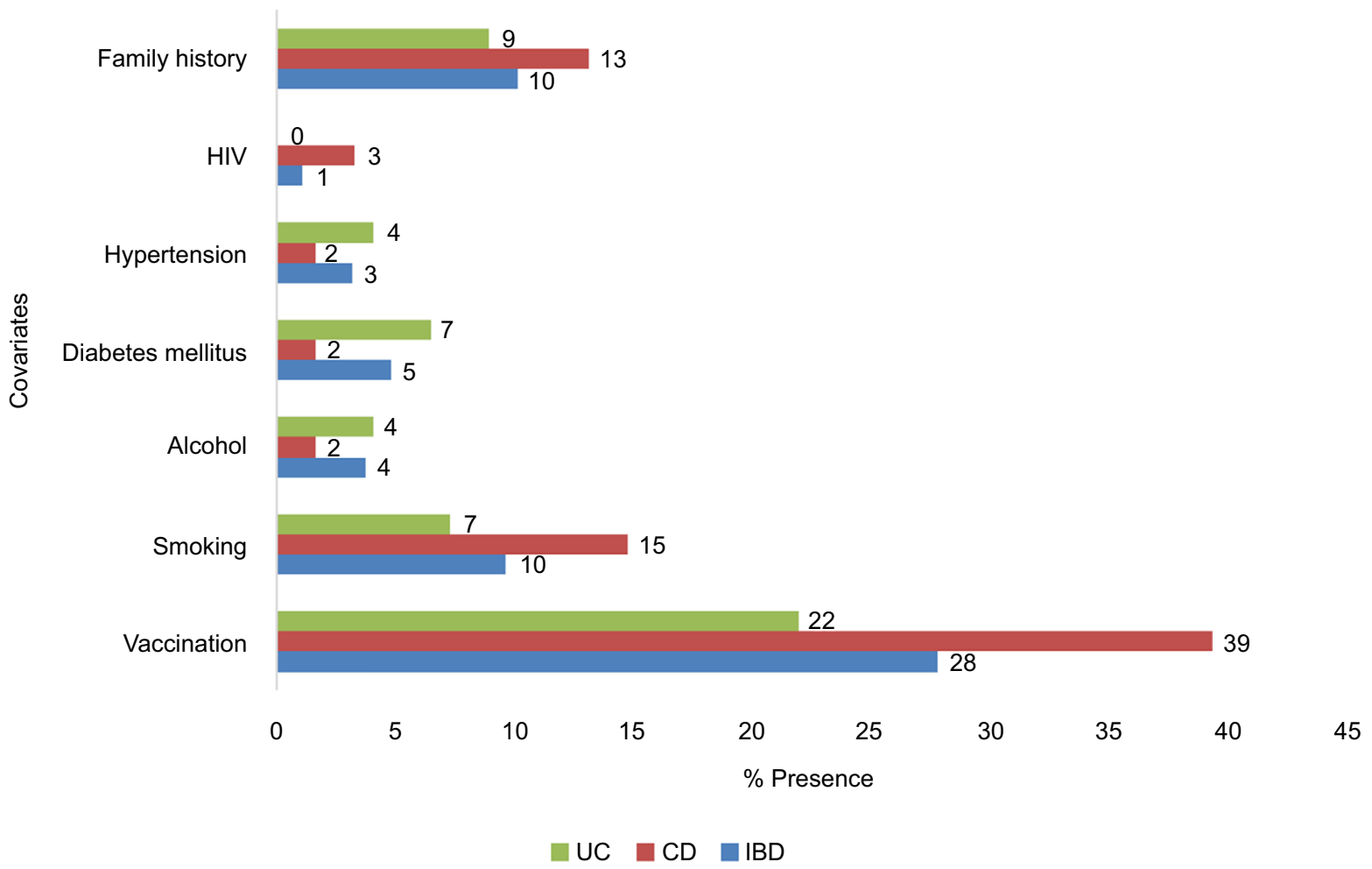

Figure S3 Family history and vaccination are the two covariates that occur the most with IBD, in particular with CD.

Abbreviations: $C D$, Crohn's disease; UC, ulcerative colitis; IBD, inflammatory bowel disease.

A

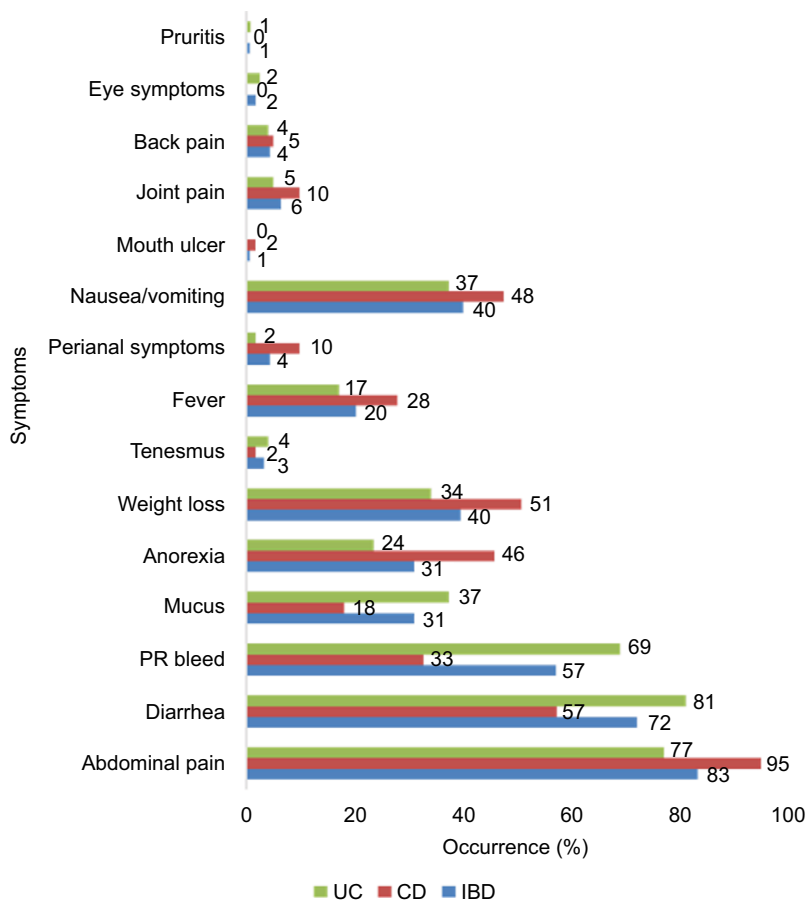

B

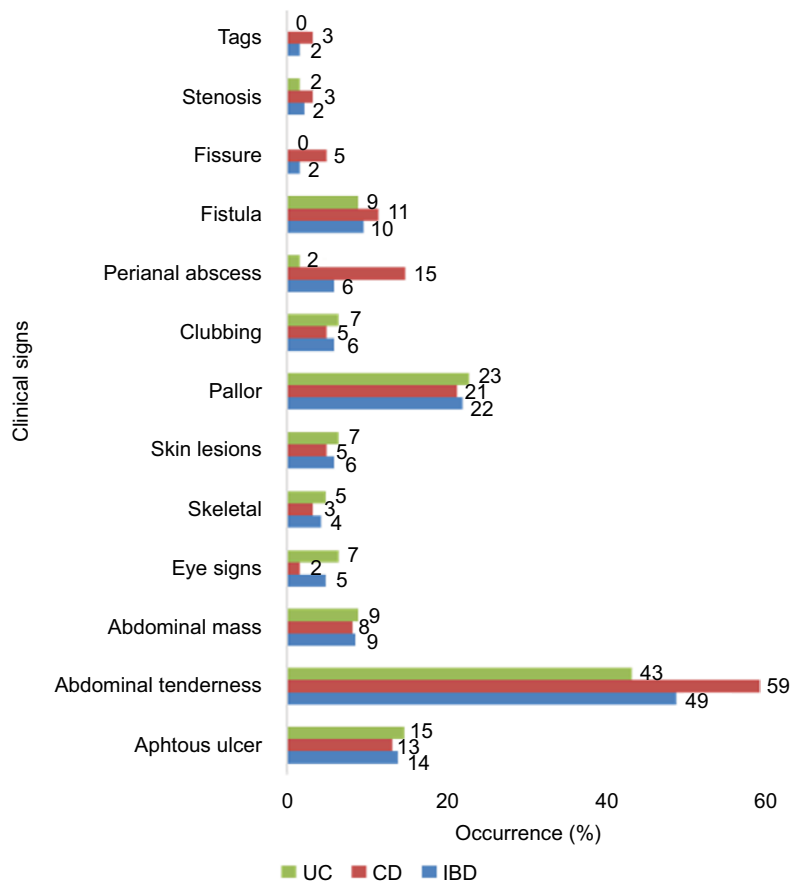

Figure S4 The percentage occurrence of a range of symptoms $(\mathbf{A})$ and clinical signs $(\mathbf{B})$ are depicted.

Notes: (A) Gastrointestinal symptoms such as abdominal pain, diarrhea, PR bleed, and nausea/vomiting are among the symptoms with the highest percentage occurrence. (B) Abdominal tenderness is reported to be the clinical sign with the highest percentage occurrence in CD, UC, and IBD.

Abbreviations: CD, Crohn's disease; PR, per rectal; UC, ulcerative colitis; IBD, inflammatory bowel disease. 
A

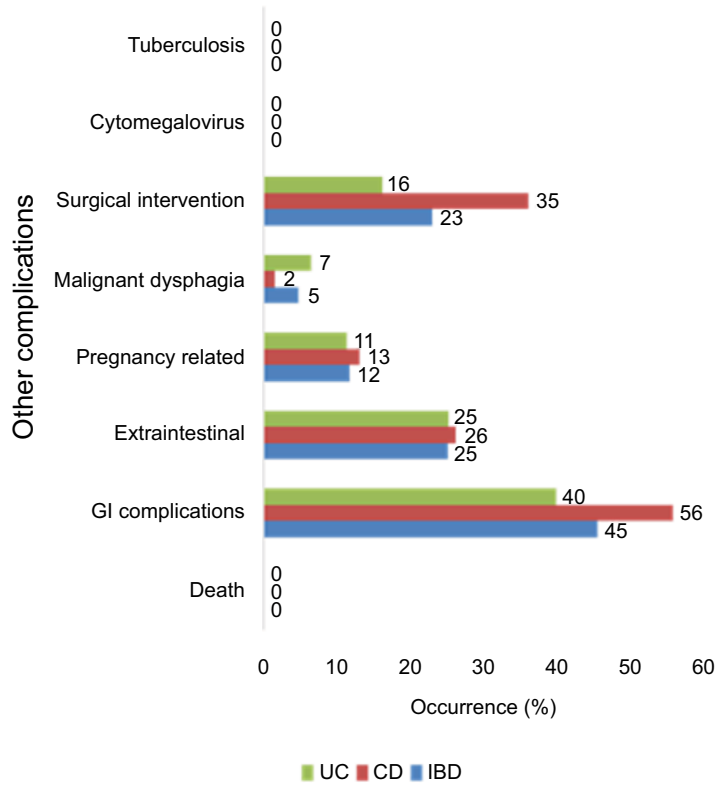

B

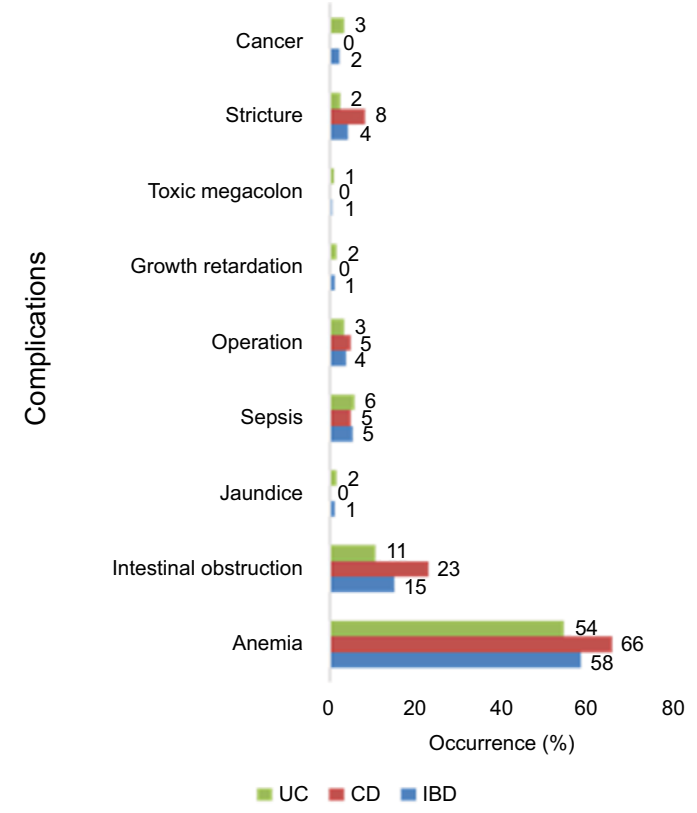

C

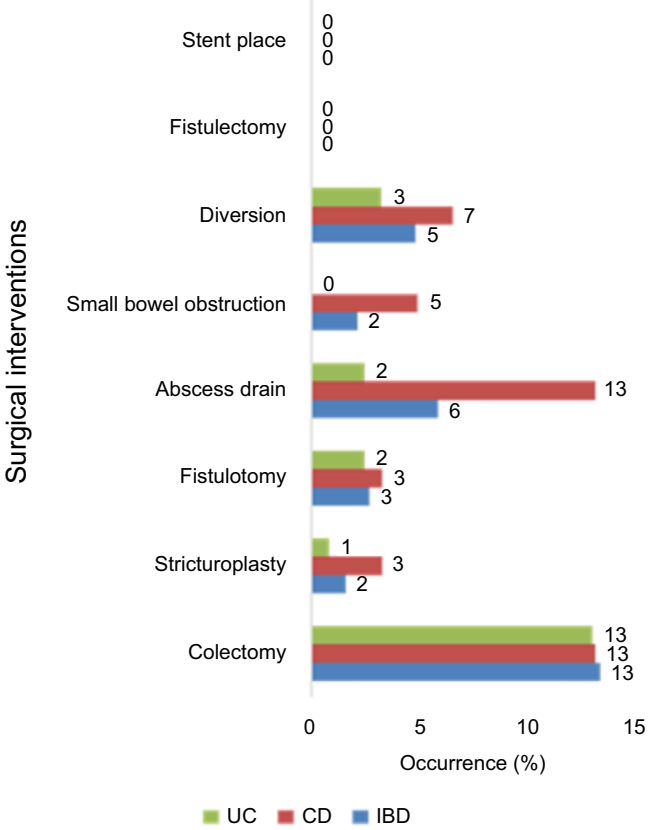

Figure S5 Analysis of complications and surgical interventions.

Notes: (A) Reported complications, (B) the type of complications was also recorded, and (C) colectomy for IBD and abscess drain for CD were revealed as the surgical interventions that occurred with the highest frequency.

Abbreviations: CD, Crohn's disease; GI, gastrointestinal; IBD, inflammatory bowel disease; UC, ulcerative colitis. 
A

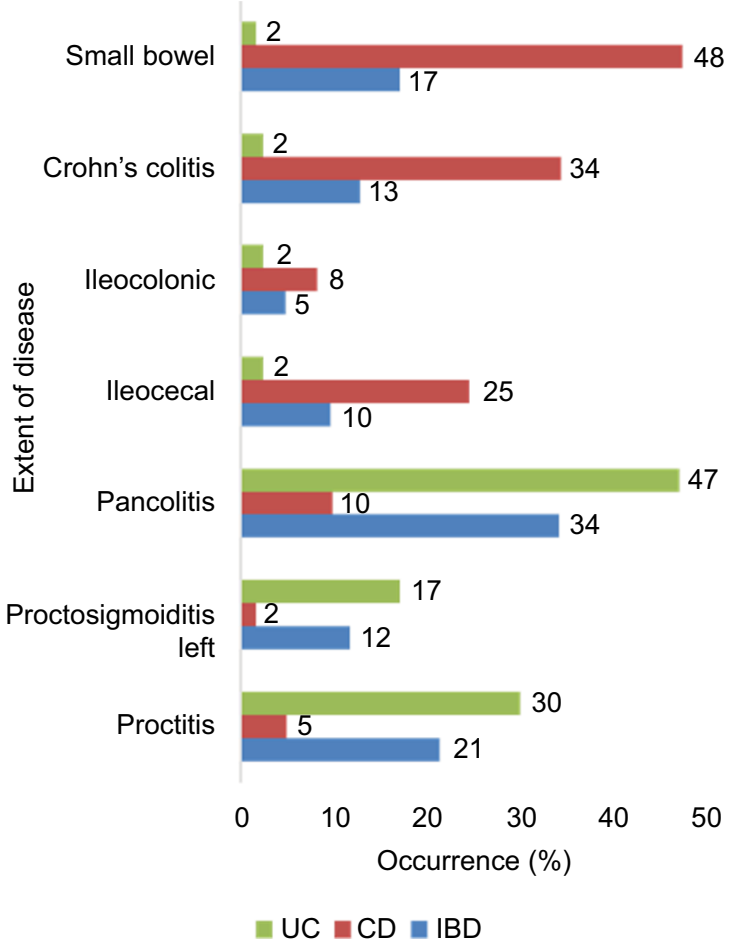

B

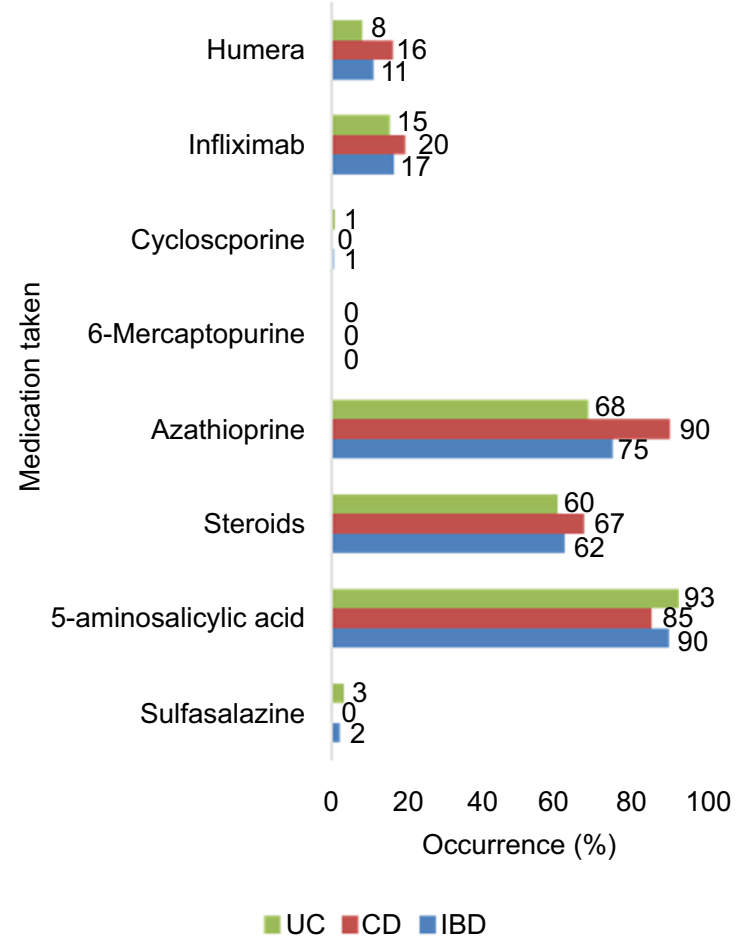

Figure S6 Extent of disease and medications taken. The extent of disease was investigated.

Notes: (A) The frequency of small bowel involvement in CD was high at $48 \%$ and Crohn's colitis in $34 \%$. Proctitis was noted in $30 \%$ of UC patients, while pancolitis was more frequently detected (47\%). (B) Azathioprine, steroids, and 5-aminosalicylic acid are the most commonly used drug treatments for IBD in Bahrain.

Abbreviations: CD, Crohn's disease; IBD, inflammatory bowel disease; UC, ulcerative colitis.

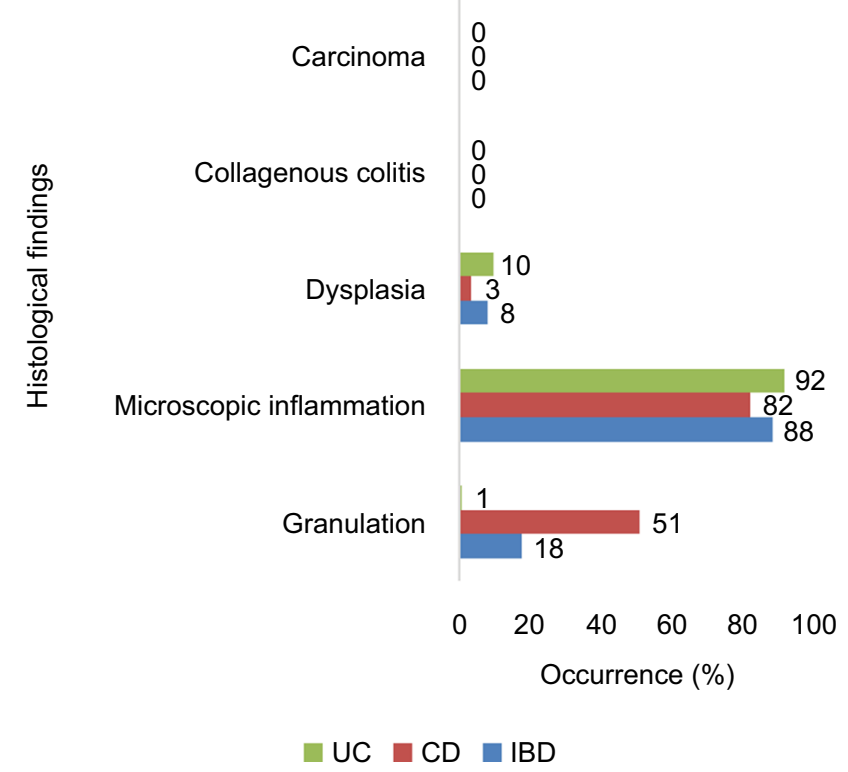

Figure S7 Microscopic inflammation represented the most common histological finding, and it was recorded in $92 \%$ of UC and $82 \%$ of CD patients.

Notes: Granulation occurred at a much higher frequency in CD patients (51\%), in comparison to UC patients (I\%). Dysplasia, collagenous colitis, and carcinoma were rare or not present in these IBD samples.

Abbreviations: CD, Crohn's disease; IBD, inflammatory bowel disease; UC, ulcerative colitis. 


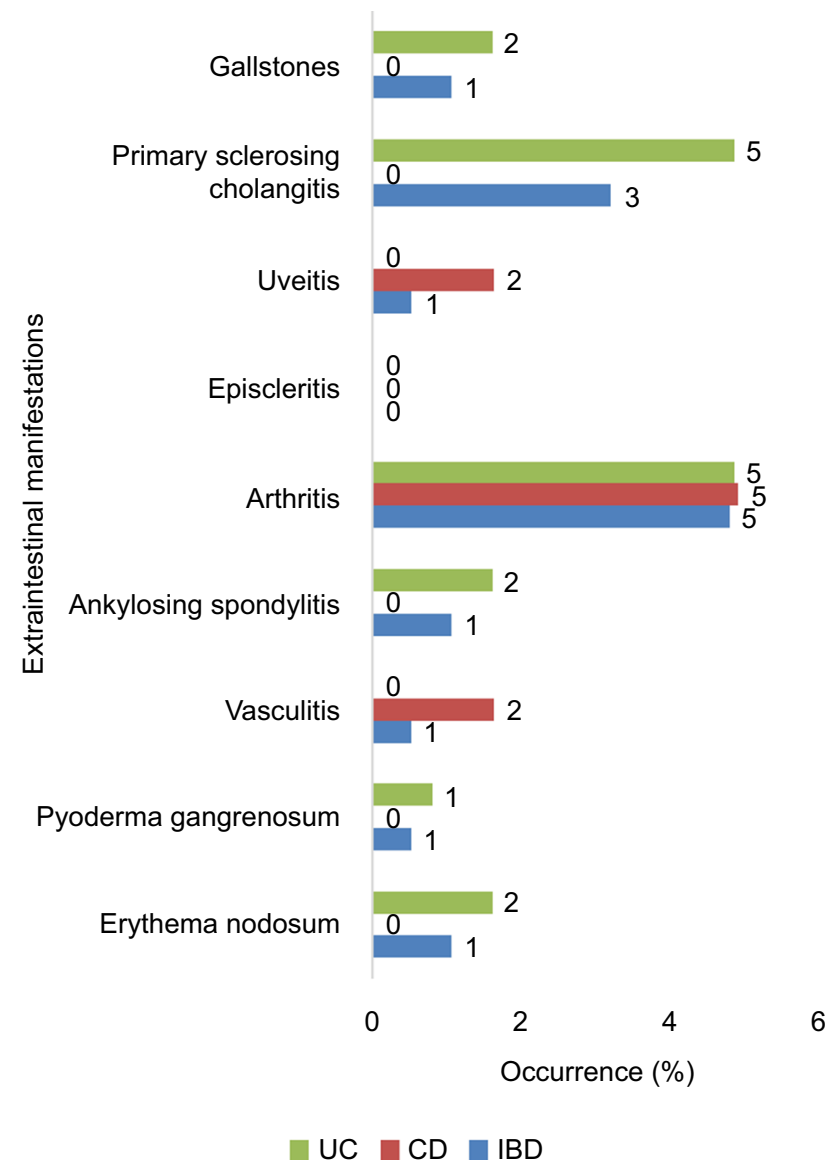

Figure $\mathbf{S 8}$ Occurrence of extraintestinal manifestations.

Notes: Arthritis occurred in $5 \%$ of IBD patients (UC and CD), and primary sclerosing cholangitis occurred in $5 \%$ of UC patients. All other extraintestinal manifestations occurred at a frequency lower than $5 \%$.

Abbreviations: CD, Crohn's disease; IBD, inflammatory bowel disease; UC, ulcerative colitis.

\section{Publish your work in this journal}

Clinical and Experimental Gastroenterology is an international, peerreviewed, open access, online journal publishing original research, reports, editorials, reviews and commentaries on all aspects of gastroenterology in the clinic and laboratory. This journal is included on PubMed. The manuscript management system is completely online

Submit your manuscript here: https://www.dovepress.com/clinical-and-experimental-gastroenterology-journal 